\title{
Characteristic time of sediment transport decrease in static armour formation
}

\author{
Berni, C., Perret, E., Camenen, B. \\ Irstea, RiverLY, centre de Lyon-Villeurbanne, 5 Rue de la Doua, CS 20244, F-69625 \\ Villeurbanne Cedex, France.
}

\begin{abstract}
A substantial laboratory data set from the literature is gathered for studying sediment transport rates under steady flow without any upstream sediment feeding. We analysed the decrease in sediment transport rate from bed armouring, i.e., coarsening of the bed surface and structure development. We quantified an equilibrium time, which corresponds to the time after which sediment transport rate is reduced by a factor of twenty. This choice is highly correlated to a characteristic time of the exponential decrease in the sediment transport rate. This equilibrium time was found to be mainly controlled by a set of four parameters: the ratio between bed shear stress and its critical value for sediment incipient motion, the Reynolds particle number, the dimensionless median diameter, and the width-to-depth ratio. The influence of each parameter can be linked to either bed mobility, armouring, or bedform development. A formula to quantify the equilibrium time as function of these four parameters is proposed. This is of great value to scale unsteady sediment transport experiments for which stepped hydrographs are very convenient and often used. A new dimensionless time is also proposed merging the decrease of sediment transport rate for hydraulically rough beds.
\end{abstract}

Keywords:

static armour, scaling, starving process, laboratory experiments, sediment transport decrease 